\title{
Demência de Alzheimer: correlação entre memória e autonomia
} Alzheimer Disease: correlation between memory and autonomy

\author{
IZABELla Dutra DE ABReu ${ }^{1}$ \\ ORESTES VicenTE FoRLENZA ${ }^{2}$ \\ Hélio LaUar de BarRos ${ }^{3}$
}

\begin{abstract}
Resumo
Discute-se neste artigo de revisão a relação entre demência e autonomia, com enfoque particular nas perdas cognitivas e no comprometimento da memória. A doença de Alzheimer (DA) é uma doença neurodegenerativa progressiva que provoca demência, comprometendo, ao longo de sua lenta evolução, a autonomia dos pacientes. A redução da autonomia é o fator determinante da dependência de um cuidador, que se torna indispensável para preservar o provimento das necessidades básicas da vida diária do paciente. É feita uma distinção entre a dependência por limitações motoras e redução de mobilidade, daquela decorrente das perdas cognitivas. Discute-se também a importância do uso racional dos instrumentos de rastreio cognitivo e avaliação funcional na mensuração do grau de autonomia dos indivíduos acometidos.
\end{abstract}

Palavras-chave: doença de Alzheimer, autonomia, qualidade de vida, memória, teste cognitivo, escala funcional.

\begin{abstract}
Alzheimer disease (AD) is a progressive neurodegenerative disorder that leads to cognitive impairment and dementia. Within a global reduction in cognitive skills, the loss of memory-related functions has a pivotal role in the development of functional disability. As opposed to physical handicap, disability due to cognitive impairment is a strong determinant of reduced autonomy, which in turn determines the degree of dependency on a caregiver. We further discuss the role of functional and cognitive screening instruments in the assessment of the degree of autonomy in patients with dementia.
\end{abstract}

Key words: Alzheimer's disease, autonomy, quality of life, memory, cognitive test, functional assessment scales.

Recebido: 19/12/2004 - Aceito: 28/02/2005

1 Médica Psiquiatra do Instituto Aurus, Residência em Psiquiatria pelo Instituto Raul Soares - FHEMIG (Fundação Hospitalar do Estado de Minas Gerais).

2 Médico Psiquiatra. Doutor em Medicina pelo Departamento de Psiquiatria da Faculdade de Medicina da Universidade de São Paulo. Médico Pesquisador do LIM-27 do Instituto de Psiquiatria do Hospital das Clínicas da Faculdade de Medicina da Universidade de São Paulo. 3 Preceptor Chefe da Residência de Psiquiatria do Instituto Raul Soares - FHEMIG, Mestre em Psicologia pela UFMG, professor da PUC Minas, psicanalista da Escola Brasileira de Psicanálise.

Endereço para correspondência: Instituto Aurus: Rua Gonçalves Dias-, 2565, Bairro Santo Agostinho - 30140-092 - Belo Horizonte - MG; e-mail: izabelladutra@yahoo.com.br 


\section{Introdução}

Demência é uma síndrome caracterizada pelo declínio progressivo e global das funções cognitivas, na ausência de um comprometimento agudo do estado de consciência, e que seja suficientemente importante para interferir nas atividades sociais e ocupacionais do indivíduo. $\mathrm{O}$ diagnóstico de demência exige a constatação de deterioração ou declínio cognitivo em relação à condição prévia do indivíduo (Corey-Bloom et al, 1995).

Dentre os critérios clínicos mais utilizados para o seu diagnóstico incluem-se o comprometimento da memória e, ao menos, um outro distúrbio como apraxia, agnosia e afasia. Tal declínio interfere nas atividades da vida diária e, portanto, na autonomia do indivíduo. A abordagem do paciente com demência deve incluir, sempre, a avaliação e monitoramento das habilidades cognitivas, da capacidade para desempenhar atividades da vida diária, do comportamento e da gravidade global do quadro (Almeida e Nitrini, 1998; Forlenza e Caramelli, 2000).

A demência se coloca como uma das maiores causas de morbidade entre idosos e sua prevalência está entre $2 \%$ e $25 \%$ dos pacientes com 65 anos ou mais (Fratiglioni e Amaduci, 1990). A doença de Alzheimer (DA) é a causa mais comum de demência no idoso, com apresentação clínica e patológica bem definidas (Marinho et al., 1997), afetando pelo menos $5 \%$ dos indivíduos com mais de 65 anos e $20 \%$ daqueles com mais de 80 anos (Jorm e Jacomb,1987).

O impacto desastroso da doença sobre a vida dos pacientes e de seus familiares é acrescido de enorme custo financeiro para a sociedade (WHO,1997). Atualmente, os gastos com serviços de enfermagem nos Estados Unidos chegam a 20 bilhões de dólares anuais com cuidados destinados a pacientes portadores de demência. Além dos gastos relacionados ao tratamento, a importância das demências no contexto farmacoeconômico é ilustrada pelo fato de que mais de 50 milhões de dólares são gastos anualmente em pesquisas sobre demência, apenas nos Estados Unidos (Costa,1998).

Os primeiros sintomas da DA aparecem usualmente após os 65 anos. Nos estágios iniciais da doença, o paciente demonstra dificuldade em pensar com clareza, tende a cometer lapsos e a se confundir facilmente, além de apresentar queda em seu rendimento funcional em tarefas complexas. Observa-se tendência ao esquecimento de fatos recentes e dificuldade para registrar novas informações. À medida que a doença progride, o paciente passa a ter dificuldades para desempenhar as tarefas mais simples, como utilizar utensílios domésticos, ou ainda para vestir-se, cuidar da própria higiene e alimentar-se (Laks,1993). Na doença mais avançada, o indivíduo acaba por perder a capacidade de funcionar de modo independente, tornando-se dependente de um cuidador. O quadro se agrava quando o paciente desenvolve sintomas psicó- ticos ou alterações comportamentais, muitas vezes disruptivas, impondo grande desgaste para o próprio paciente e sobrecarga ao cuidador. O óbito geralmente advém após dez a 15 anos de evolução, como complicação de comorbidades clínicas ou quadros infecciosos, em indivíduos que se tornaram progressivamente fragilizados pela doença crônica (Engelhardt et al, 2001). Em cada uma destas etapas sucessivas, pode-se observar gradativa perda da autonomia, e conseqüente aumento das necessidades de cuidados e supervisão de terceiros para os portadores da doença (Laks,1997).

Com o progresso das práticas médicas, levando à maior eficácia das intervenções e tratamentos, a mortalidade populacional tem-se associado, cada vez mais, às condições próprias do envelhecimento e às doenças degenerativas (Levcovitz,1999). O impacto subjetivo desta relação de freqüência e ocorrência gera sentimentos de impotência, desamparo, fragilidade e falta de perspectiva de futuro entre os portadores destes processos degenerativos e seus familiares. As manifestações e as conseqüências de DA têm sido popularizadas em função do crescente interesse dos veículos de comunicação. Observando-se e escutando os pacientes portadores de doenças degenerativas e seus familiares, encontramos com freqüência a expressão "morte em vida" para definir uma nova modalidade de velhice. Aqui, processos mórbidos degenerativos aceleram a decadência psíquica e funcional do indivíduo, comprometendo sua qualidade de vida e apresentando uma modalidade de morte que destrói o sujeito, sem, contudo, ceifar, efetivamente, a sua vida. Neste estudo de revisão, abordaremos alguns instrumentos de avaliação de pacientes com DA que enfocam atividades diárias e cognitivas e, por este viés, traçar uma correlação entre autonomia e memória.

\section{A magnitude do comprometimento cognitivo: instrumentos de avaliação cognitiva e funcional}

Os instrumentos de rastreio que identificam casos leves de demência têm sido importante objeto de estudo. Pesquisadores sugerem que a combinação de uma escala funcional, que avalia atividades da vida diária e um teste cognitivo (por exemplo, o Mini-Exame do Estado Metal [MEEM]) seria complementar em indivíduos com suspeita de síndrome demencial e, como conseqüência, aumentariam a sensibilidade e especificidade para rastrear a doença em uma população não homogênea como a nossa, do ponto de vista cultural e socioeconômico (Mackinnon e Mulligan, 1998).

Dentre as múltiplas escalas de avaliação funcional existentes, discutiremos a seguir: (1) o Questionário do Informante sobre o Declínio Cognitivo do Idoso (Informant Questionnaire of Cognitive Decline in the Elderly [IQ-CODE]) (Jorm e Jacomb,1989); (2) a Escala 
Bayer de Atividades da Vida Diária (B-ADL) (Hindmarch et al.,1998); e (3) o Índice de Katz (Gorestein, 2000).

O IQ-CODE avalia cognição entrevistando cuidador ou alguém que tenha contato próximo ao paciente. São 26 questões que avaliam o desempenho atual em diferentes situações da vida diária comparando com o desempenho anteriormente observado ao longo de um continuum de dez anos. A pontuação é feita segundo a impressão de desempenho "muito melhor", "melhor", "sem mudanças significativas", "pior" ou "muito pior", com escores, respectivamente, de 1, 2, 3, 4 ou 5 . Os escores brutos variam entre 26 e 130 , sendo que a média aritmética desse escore pelo número de questões respondidas dá origem a um escore, que pode ser comparado com pontos de corte previamente estabelecidos (Bustamante et al., 2003). Sua validade reflete um declínio cognitivo prévio, prevê quadros demenciais e se correlaciona com vários testes cognitivos; não sofre influência de escolaridade.

A B-ADL avalia desempenho atual do paciente em atividades da vida diária. $\mathrm{O}$ cuidador ou alguém próximo ao paciente deve responder a 25 questões em uma escala que varia entre 1 e 10. Existe a possibilidade "não sabe" e "não se aplica", quando não se tem a informação ou quando a dificuldade se relaciona a um outro transtorno não-cognitivo, respectivamente. Tais perguntas serão excluídas do número de pontos totais. O estudo de Bustamante et al. (2003) pontua que a média superior nesta escala, seria mais bem explicada pela boa performance em indivíduos com alta escolaridade. Utilizada em diferentes populações, permite detectar casos leves de demência, bem como, ao ser associada ao MEEM apresenta sensibilidade de $86,7 \%$ para diagnosticar a doença. Em estudo realizado na Espanha, Alemanha e Reino Unido, foram avaliados 1.433 pacientes com declínio cognitivo, e o resultado nos três países foi similar, testando, assim sua validade em línguas e culturas diferentes (Erzigkeit et al., 2001).

O Índice de Katz é uma escala mais descritiva que avalia desempenho em atividades da vida diária, ou seja, sua capacidade funcional e as divide em rotineiras (tomar banho, vestir-se, ir ao banheiro, continência e alimentação) e instrumentais (usar telefone, preparar comida, fazer compras, locomoção fora de casa, medicação e dinheiro). Classifica os pacientes em independente (I), dependente (D) e necessitado de assistência (A). Também respondida pelo cuidador ou pelo paciente. Tal escala não tem um ponto de corte específico.

Segundo Bustamante et al. (2003), em seu estudo sobre combinação de instrumentos para avaliar DA, o MEEM classificou corretamente $86,8 \%$ dos pacientes; o IQ-CODE $89,5 \%$ e a B-ADL $92,1 \%$. Além disso, a combinação de um teste cognitivo, como MEEM, e uma escala funcional (IQ-CODE e B-ADL) proporcionam maior precisão diagnóstica, sem aumentar substancialmente o tempo de avaliação.
Os questionários respondidos pelos informantes sofrem influência de fatores não-cognitivos, tais como o estado afetivo do paciente e do informante e o grau de proximidade entre eles. Segundo Loewenstein (1995), os familiares fornecem respostas mais acuradas no que tange a capacidade funcional do paciente, mas podem sub ou superestimá-la, de acordo com sua percepção do problema, o grau de estresse e sobrecarga, ou até mesmo suas características de personalidade e capacidade cognitiva.

Existem também testes denominados "ecológicos", que se aproximam de situações cotidianas do paciente. Como exemplos, o teste de memória lógica de Wechsler (1987) que avalia memória verbal e visual, retenção, evocação média e tardia, e o teste comportamental de Rivermead (Wilson et al.,1985), que enfatiza tarefas com relevância prática, simulando situações rotineiras, como lembrar nome e sobrenome de pessoas apresentadas por fotografia, lembrança de pontos principais de uma estória, reconhecimento de objetos entre outros distratores e de faces de pessoas. Tais testes têm a importância de simular o dia-a-dia do paciente, e são menos influenciados por viés cultural.

Entre os testes cognitivos mais comumente utilizados no manejo clínico de pacientes com demência, podemos citar o MEEM (Folstein et al., 1975), o Teste Cognitivo de Cambridge (CAMCOG) (Roth et al, 1986), a Escala de Blessed para demência (Blessed et al,1968), e a Escala para Avaliação da Doença de Alzheimer (ADAS-COG)(Mohs e Cohen,1988). Todos esses instrumentos sofrem considerável influência do grau de escolaridade e habilidades prévias dos pacientes, devendo ser validados para cada contexto sociocultural. Ainda, esses instrumentos requerem alguma preservação das capacidades sensório-motoras e de linguagem, bem como o contato direto entre o paciente o profissional que as aplica.

O MEEM é o teste de rastreio e triagem mais utilizado no mundo, pois é simples, de aplicação rápida e auto-explicativa. Determina a extensão da avaliação cognitiva subseqüente à sua aplicação em sujeitos com demência moderada e severa. É composto por diversas questões, caracteristicamente, agrupadas em sete categorias, cada uma com a finalidade de avaliar funções cognitivas específicas como orientação, retenção ou registro de dados, atenção e cálculo, memória e linguagem. $\mathrm{O}$ escore do MEEM pode variar de um mínimo de zero a 30 pontos. Tombauugh e McIntyre (1992) mostraram que a escala tem boa consistência interna e confiabilidade teste-reteste $(0,8 \%$ a $0,95 \%)$, e que o ponto de corte 23/24 tem boa a excelente sensibilidade (54\% a $100 \%)$ e especificidade $(62 \%$ a $100 \%)$ para zero diagnóstico de demência em indivíduos com alta escolaridade.

No Brasil, O MEEM foi traduzido e adaptado por Bertolucci et al. (1994). Nesse estudo, que se tornou uma referência obrigatória em nosso meio, foi demons- 
trada a importância da escolaridade no escore total do teste. O teste foi aplicado em 530 indivíduos, classificados segundo suas idades e escolaridades e constatouse que ao comparar os quatro níveis de escolaridade (analfabetos, baixa, média e alta) foram obtidas diferenças estatisticamente significativas $(p<0,0001)$ entre os escores, exceto para os indivíduos de baixa e média escolaridade quando comparados entre si. Portanto, foram estipulados valores de corte para cada grupo, evitando-se assim erros diagnósticos, tomando como referência o percentual $5 \%$ da porção inferior da curva de distribuição de valores dos escores. Os pontos de corte para o MEEM segundo o nível de escolaridade em nosso meio são: 13 pontos para analfabetos, 18 para escolaridade média (até 8 anos de instrução formal) e 26 para indivíduos de alta escolaridade (mais de 8 anos).

Embora, não substitua uma avaliação completa e seja pouco sensível para detectar variações sutis na cognição, o MEEM é amplamente utilizado pelos profissionais da área. Almeida e Nitrini (1998) investigaram a sensibilidade e especificidade de diferentes pontos de corte do MEEM para o diagnóstico de demência em uma amostra de 211 idosos atendidos em ambulatório. Observou-se a sensibilidade de $84 \%$ e especificidade de $60 \%$ para os pontos de corte $23 / 24$. Tal estudo também constatou que, quanto mais jovem e maior o nível educacional do indivíduo, melhor o desempenho no MEEM.

As baterias neuropsicológicas constituem-se em seqüências de testes que avaliam comportamento e cognição. Podem ser padronizadas (compostas pelos mesmos testes) ou flexíveis (compostas por testes agrupados de acordo com a necessidade). São importantes para elaboração de planos de reabilitação, políticas de prevenção de saúde mental (Malloy-Diniz e Carvalho, 2001). Além disso, facilitam o estabelecimento de perfil cognitivo basal antes, durante e depois de tratamentos, bem como colaboram para o diagnóstico diferencial em condições que envolvam prejuízo cognitivo.

A bateria CERAD (Morris et al.,1993) inclui avaliação da memória (fixação, evocação e reconhecimento), linguagem (nomeação e fluência verbal), praxia (cópia de desenhos geométricos), função executiva (teste das trilhas), além de um teste geral (MEEM). Em nosso meio, os testes dessa bateria mais sensíveis para o diagnóstico de demência foram o MEEM $(97,6 \%)$ e fixação da memória verbal (85,7\%), e os mais específicos foram nomeação $(69,4 \%)$ e fixação da memória $(87 \%)$. O melhor equilíbrio (acima de $75 \%$ nos dois aspectos) foi para os testes de fixação e evocação da memória. Estes resultados indicam que a adaptação foi adequada e fornecem um padrão para o desempenho esperado em relação às demências (Bertolucci et al., 2001). Algumas vantagens da bateria CERAD, em relação a outros instrumentos de rastreio são: a possibilidade de comparar resultados entre diferentes grupos e estudos, a alta concordância dos resultados entre os clínicos e a melhor sensibilidade para a detecção de quadros demenciais em quadros iniciais.

\section{Memória e autonomia em indivíduos idosos}

Acompanhando os testes que avaliam as demências podemos perceber que, apesar do acometimento de muitos aspectos da cognição, a memória tem destaque especial nesse processo.

A DA é caracterizada pelo déficit na memória episódica de longo termo (devido à baixa performance na evocação) e na memória de curto prazo que pode estar relacionada com a gravidade do quadro (Izquierdo, 2002). Percebemos que pacientes com DA desempenham baixa atividade em tarefas que envolvem nomeação, fluência verbal e uso correto de sentenças e vocabulários, todos estes utilizados para formar a memória semântica, mais bem definida como uma representação mental de palavras, objetos e significação de ações para memória de longo prazo. Assim, ações para realizar atividades da vida cotidiana estariam comprometidas, quer por esquecimento, quer por déficit no próprio conceito da ação em si (Grossman et al.,1997).

A perda da memória dificulta a aproximação das pessoas em suas relações afetivas, sociais e familiares (Rozenthal et al, 1995). A memória biográfica dá o reconhecimento da identidade. Sem lembrar-se de fatos, de lugares e de pessoas, diz-se que há menos da pessoa a cada dia; a mesma fica impossibilitada de se relacionar, cuidar de si, planejar sua qualidade de vida; perde sua razão, autonomia e coerência. Dá-se a impressão que o eu se desvincula das funções cognitivas, garantindo sua sobrevivência apenas.

A noção de autonomia, no que tange a interdependência desta com uma memória íntegra, reside na capacidade individual de cuidar de si mesmo, executar tarefas que lhe permitem a adaptação psicossocial, e ser responsável pelos próprios atos.

As avaliações cognitivas realizadas em pacientes idosos demonstram que a senilidade, com ou sem demência, produz alterações cognitivas, especialmente de memória de fixação que determina perda na capacidade executiva. Esta situação gera, em grau variável, dependência do sujeito a um terceiro, nomeado cuidador. Seria essa dependência, sinônimo de perda de autonomia? Esta relação de dependência do sujeito ao seu cuidador merece avaliações quantitativas e qualitativas. As escalas de avaliação costumam ordenar a observação do comprometimento da capacidade de cuidar de si mesmo e de executar tarefas cotidianas. Há evidente intenção de hierarquizar como mais grave a perda da capacidade de se auto-cuidar. O cruzamento dos resultados destas escalas funcionais com avaliações minuciosas da capacidade cognitiva pode indicar que a dependência pode ser mais bem caracterizada cognitivamente. Assim sendo teremos, supostamente, duas categorias distintivas: um grupo 
como perda da capacidade de se cuidar, mas com preservação cognitiva, e outro grupo com perda da capacidade de se cuidar e com deterioração da capacidade cognitiva. Este exercício de categorização permite hipotetizar uma diferença qualitativa entre aqueles que se mostram dependentes de um terceiro. Supostamente a dependência no primeiro grupo preserva pontos essenciais na autonomia, ou seja, capacidade de falar, pensar e se responsabilizar pelos seus atos entendidos aqui como expressão de desejo e não como ação motora. Atribuímos, assim, para a noção de autonomia uma maior aptidão cognitiva do que motora, e deixamos para a dependência, a noção de perda da capacidade motora, ainda que em extremos elas possam figurar como sinônimos.

Nestes termos podemos sustentar que autonomia pode ser incluída no eixo dependência-independência. Quanto maior for o grau de independência maior a probabilidade de autonomia, mas, em condições de dependência parcial, o sujeito ainda assim pode ter sua autonomia preservada dependendo dos arranjos sociais que ele for capaz de fazer. O paradoxo dependência-autonomia não deve ser considerado, a princípio, como contradição; e sim, como oposição não excludente. Arranjos sociais no contexto dos contratos sociais podem viabilizar aumento da qualidade de vida e conseqüente aumento da autonomia.

Na velhice, a manutenção da qualidade de vida está intimamente ligada à autonomia (Leibing,1997). Uma forma de procurar quantificar a autonomia, neste nível, é medir índices de qualidade de vida de um indivíduo. Esta qualidade de vida pode ser indicada pela capacidade que o idoso tem de desempenhar as funções necessárias à manutenção da sua vida diária e prática, de modo a torná-lo independente dentro do seu contexto socioeconômico e cultural. As escalas funcionais e cognitivas nos fornecem tais dados.

Se aceitarmos a definição de demência como deterioração cognitiva e intelectual associada à redução da capacidade funcional global de um indi- víduo, surge a questão de como avaliar a qualidade de vida de forma acurada e mensurável, através não somente da capacidade de cuidar de si de modo dependente ou independente de arranjos sociais contratuais, mas sim através da disponibilidade psíquica de desenvolver tais tarefas e compreender o sentido das mesmas, de modo adaptativo e inventivo, segundo exigências situacionais externas e da motivação interior. Estamos, portanto diante de um novo eixo de avaliação da autonomia, que pode ser formulado a partir de avaliações cognitivo-executivas. Entendendo a cognição como força ou potência de eleição e discriminação para ações executivas dirigidas e voluntárias.

O termo "função executiva" indica habilidade cognitiva e o princípio de organização necessário para lidar com as situações flutuantes e ambíguas do relacionamento social. Inclui formulação de objetivos e de conceitos, motivação, planejamento, auto-regulação, insight, abstração, análise, manipulação de conhecimentos adquiridos e flexibilidade mental. São funções psíquicas fundamentais para a produção de respostas comportamentais adequadas aos diferentes contextos, e que se encontram prejudicadas nos quadros demenciais.

Como já citado, há um modelo cuja avaliação do rendimento funcional do idoso está focalizada na análise de características pessoais, da tarefa em si, e do contexto em que esta se insere. Muitos déficits funcionais não envolvem somente a incapacidade de desempenho, mas a inabilidade de iniciar ações voluntárias. Nesses casos, utilizamos o termo "memória prospectiva" (Eistein e McDaniel, 2002). Falhas nesta memória resultam, portanto, na incapacidade em iniciar uma seqüência de ações que são demandadas pelo ambiente e que estão fortemente relacionadas, com atividades da vida cotidiana, e autonomia própria do sujeito. Sendo assim, concluímos que a memória, como função central nos processos cognitivos, tem relação direta com atividades que afetam a autonomia do indivíduo.

\section{Referências bibliográficas}

Almeida, O.P. Nitrinı, R. - Demência. São Paulo: Fundação Byk, 1998.

BertolucCl, P.H.F.; BRUCKI, S.M.D.; CAmpaccl,S.R.; Jullano,Y. - 0 Mini Exame do Estado Mental em uma população geral: impacto da escolaridade. Arq Neuropsiquiatria 1994;52:1-7.

Bertoluccl, P.H.F.; Oкамoto, I.; Brucki, S.; Siviero, M.; Neto, J.; Ramos, L. - Applicability of the CERAD neuropsychological battery to brazilian elderly. Separata. Arq Neuropsiquiatria 2001;59(3-A):532-6.
BLESSED, G.; ToMLISON, B.E.; RоTH, M. - The association between quantitative measures of dementia and of senile change in the cerebral grey matter of the elderly subjects. Psychiatry 1968;114:797-811.

Bustamante, E;;Bottino, C.; LoPES, M. etal. - Instrumentos combinados na avaliação de demência em idosos: resultados preliminares. Arq Neuropsiquiatria 2003;61(3-A):601-6.

Corey-Bloom, J.; Thal, L.; Galasko, D.; Folstein, M.; Drachman, D.; RASKIND, M.; LANSKA, D.J. - Diagnosis and evaluation of dementia. Neurology 1995;45:211-8. 
Costa, L.V.A. - Política nacional do idoso: perspectiva governamental. Anais do I Seminário Internacional do Envelhecimento Populacional: Uma agenda para o fim do século. MPAS, Brasilia, 1988.

Eistein, G.0.; McDanieL, M.A. - Normal ageing and prospective memory. Journal of Experimental Psychology: Learning, Memory and cognition. 1990;16,717-26. In: Leibing, A.; Scheinkman, L. - The diversity of Alzheimer Disease: Different approaches and contexts. IBUP, 2002a.

Eistein, G.0; McDaniel, M.A. - Remembering to do things: Remembering a forgotten topic. In: Leibing, A.; Scheinkman, $\mathrm{L}$. The diversity of Alzheimer Disease: Different approaches and contexts. IBUP, 2002b.

Engelhardt, E.; Pereira, E.R.F.; Santos, M.D.; Cavalcanti, J.L.S.; Temponi, G.M.; Albuduerque, E. - Galantamina no tratamento da DA: da pesquisa ao paciente. Revista brasileira de Neurologia 2001;37(2):6-16.

ERzIGKeit, H.; Lehfeld, H.; Pena-Casanova, J. et al. - The Bayer activities in daily living scale: results from validation study in three European countries. Dement Geriatr Cogn Disord 2001;12:348-58.

Folstein, M.F;; Folsteln, S.E.; McHugh, P.R. - “Mini Mental state”. A practical method for grading the cognitive state of patients for the clinician. J Psychiatr Res 1975;12:189-98.

Forlenza, O.V.; Caramelli, P. - Neuropsiquiatria Geriátrica. São Paulo: Atheneu, 2000.

Fratiglioni, L.; Amaducl, L. - An epidemiological survey of Alzheimer desease. In: Battistin, L.; Gesterbrand, F. (Eds.). Aging brain and dementia. New York: Wiley-Liss, 1990, pp.16-29.

Gorenstein, C.; AndRade, L.H.S.G.; ZuARDI, A.W. - Instrumentos para avaliação de pacientes com Demência. (Ed.1) Escalas de Avaliação Clínica em Psiquiatria e Psicofarmacologia. São Paulo: Lemos, 2000, pp. 331-43.

Grossman, M.; Mickanin, J.; ONISHI, K.; Robinson, K.; D'ESPOSIto, M.B. - Lexical Acquisition in Probable Alzheimer's Disease. Brain and Language 1997;60:443-63.

Hindmarch, l.; Lehfeld, H.; Pena-Casanova, J. et al. - The BayerActivities of daily living scale (B-ADL). Dement Geriatr Cogn Disord 1998;9(Suppl. 2):20-6.

Izouierdo, I. - Memória. Capítulo 2. São Paulo: Artmed, 2002. JoRm, A. F.; JACOMB, P.A. - The informant questionnaire on cognitive decline in the elderly (IOCODE): Sociodemographic correlates, reliability,validity and some norms. Psychol Med 1989;19:1015-22.

Jorm, A.F.; Korten, A.E.; Henderson, A.S. - The prevalence of dementia: a qualitative integration of the literature. Acta psychiatrica Scandinavica 1987;76:465-79.

LaKs, J. Revista Brasileira de Neurologia 1997;33(4):201-6.
LeIBING, A. - Antropologia de uma doença orgânica: doença de Alzheimer e fatores culturais. In: Cadernos IPUB, Instituto de Psiquiatria da UFRJ (Ed.): Envelhecimento e Saúde Mental - Uma Aproximação Multidisciplinar, 1997;10:157-74.

Levcovitz, E.C. - Morte do corpo e morte do eu: a interdição da morte na doença de Alzheimer. (Dissertação de Mestrado em Psicanálise) - IPUB, Rio de Janeiro, 1999.

Loewenstein, D.A.; Rubert, M.; Arguelles, T.; Duara, R. Neuropsychological test performance and the prediction of functional capacities among Spanishspeaking and English - speaking patients with dementia. Arch Clin Neuropsychol 1995;10(2):75-88.

Mackinnon, A.; Mulugan, R. - Combining cognitive testing and informant report to increase accuracy in screening for dementia. Am J Psychiatry 1998;155:1529-35.

MalloY-Diniz, L.F.; CARValho, A.M. - 0 exame neuropsicológico e suas contribuições à psiquiatria. Psiquiatria Biológica 2001;9(2):66-77.

Marinho, V.; Laks, J.; Engelhardt, E. - Aspectos neuropsiquiátricos das demências degenerativas nãoAlzheimer. Revista Brasileira de Neuropsicologia 1997;33(1):31-7.

Mohs. R.C.; Cohen, L. - Alzheimer's Disease Assessment Scale (ADAS) - Psychopharmacology Bulletin 1988;24:627-8.

Morris C.; Heyman, A.; Mohs, R.C. et al. - The consortium to establish a registry for Alzheimer's disease. Part IV. Rates of cognitive change in the longitudinal assessment of probable Alzheimer's disease. Neurology 1993:43:2457-65.

Roth, M.T.Y.M.E.; Mountuoy, C.Q.; Huppert, F.A.; Hendrie, H.; Verma, S.; GODDARD, R. - CAMDEX: a standardised instrument for the diagnosis of mental disorder in the elderly with special reference to the early detection of dementia. British Journal of Psychiatry 1986;149:698-709.

Rozenthal, M.; EngelHardt, E.; Laks, J. - Memória: aspectos funcionais. Rev Bras Neurol 1995;31(3):157-60.

Tombaugh, T.N.; McIntrre, N.J. - The Mini Mental State Examination: a comprehensive review. Journal of the American Geriatrics Society 1992;40:922-35.

WeChSLER, D. - Wechsler Memory Scale - Revised Manual. San Antonio, Texas: The Psycological Corporation, 1987.

Wilson, B.A.; CockBurn, J.; Baddley, A. - The Rivermead Behavioral Memory Test. Reading: Thames Valley Test Co, 1985.

World Health Organization - Organization of care in psychiatry of the elderly - a technical consensus statement Programme on mental health, World Psychiatric Association, Geneva, 1997. 